of rehabilitation and open-ward forensic patients (approximately $45 \%$ in each year) who often receive higher doses than general psychiatric patients. Because of our largely communitybased service, it might be speculated that those patients actually requiring hospital admission are more severely ill, reflected by higher rates of compulsory admission (Singh et al, 1998) and have greater levels of behavioural disturbance prompting prescribing of higher doses of medication but not necessarily benefiting from them.

When p.r.n. prescribing is also taken into account, this results in a statistically significant increase, both in the mean dose in chlorpromazine equivalents per day and in the number of patients who fall into the 'high-dose' category for each year. This is usually as a result of prescribing by junior (often non-MRCPsych) psychiatrists and contrary to the Consensus Statement guidelines. Although the actual dispensing of p.r.n. medication occurs infrequently (4-5\% of p.r.n. prescriptions) and suggests that the issue of injudicious prescribing is in practice less clinically relevant than initially expected, we believe that it still reflects a hidden potential for the unwitting prescription of high doses.

It is clear from the Consensus Statement that there is little evidence from controlled clinical trials for the superior effectiveness of high-dose antipsychotic medication prescribing, even for behavioural control. Therefore the addition of p.r.n. doses to already high-dose prescribing would appear to rarely have any clinical justification. We would recommend regular surveys and audit of antipsychotic prescribing and the development of local guidelines for junior staff on good prescribing practice. These guidelines should address, in particular, the area of cautious p.r.n. prescribing by juniors and monitoring by consultants. We would also encourage identification of those patients on high-dose antipsychotic medication and suggest frequent medication reviews involving pharmacists.

\section{References}

BAZIRE, S. (1997) Psychotropic Drug Directory. Dinton: Quay Books Division, Mark Allen publishing.

Cornwall, P., Hassanyeh, F. \& HoRn, C. (1996) High-dose antipsychotic medication. Improving clinical practice in a psychiatric special (intensive) care unit. Psychiatric Bulletin, 20, 676-680.

KRASUCKI. C. \& MCFARLANE, F. (1996) Electrocardiograms, high-dose antipsychotic treatment and college guidelines. Psychiatric Bulletin, 20, 326-330.

NEWTON, K., MURTHY, R. \& QURESHI, J. (1997) Antipsychotic prescribing in light of the consensus statement of the College. Psychiatric Bulletin, 21, 408-410.

PINNER, G. \& EDGAR, S. (1996) Audit of high-dose and combination antipsychotic medication prescribing. Pharmaceutical J, 256, 762-763.

Singh. S.. Croudace. T., Beck. A.. et al (1998) Perceived ethnicity and risk of compulsory admission. Social Psychiatry and Psychiatric Epidemiology. 33. 39-44.

THOMPSON, C. (1994) The use of high-dose antipsychotic medication (Consensus Statement). British Journal of Psychiatry, 164, 448-458.

TORKINGTON, B., HOGG, S., POWELl, G., et al (1994) Antipsychotic medication use in relation to BNF guidelines. Psychiatric Bulletin, 18, 375-376.

WARNER, J., SLADE, R. \& Barnes, T. (1995) Change in neuroleptic prescribing practice. Psychiatric Bulletin. 19. 237-239.

*John Milton, Senior Registrar in Psychiatry, Nottingham Healthcare NHS Trust, Duncan Macmillan House, Porchester Road, Nottingham NG3 6AA; John Lawton, Senior Pharmacist, Mark Smith, Clinical Audit Department, Nottingham Healthcare NHS Trust, Nottingham; Ann Buckley, Senior Registrar in Psychiatry, South Birmingham Mental Health NHS Trust, Mindleson Way, Birmingham

*Correspondence

\title{
Rationale behind psychiatrists' choice of drug
}

\author{
John Dickson-Mulinga
}

Aims and method The study investigated the reasons
that influence medical staffs' choice of a specific drug
over another given the same clinical situation, by use of
a questionnaire-based survey. The study population
was 88 clinicians of various ranks, at psychiatry units in
National Health Service university and district general

Psychiatric Bulletin (1998), 22, 677-680 hospitals in Greater Manchester. Responses in the factors: personal experience, scientific evidence, influence from colleagues, economic consideration, influence by drug representatives, ward or unit policy and other in choice of prescription, were the main outcome measures. 
Results Of the 546 responses generated by the 88 clinicians, 263 (48.2\%) were for personal experience as the most important influence on choice of prescription. Scientific evidence was the second most important factor with 117 (21.4\%) responses. Economic consideration and influence by drug representatives were least important. This trend held for all ranks except for registrars, where scientific evidence was as important as personal experience.

Clinical implications As prescribers, clinicians trust mostly in their own personal experience. This may not be unique to psychiatrists. An understanding of how clinicians gain 'personal experience' could be important in improving rational prescribing.

There seems to be wide variation in the prescribing habits of medical staff treating the same or similar conditions. This is supported by research, and in general practices at least, there is little evidence that this variation is justified on clinical or demographic grounds (Bateman et al, 1996). The present quality of prescribing still falls short of optimal standards (Hogerzeil, 1995). This has implications for prescribers as well as patients in terms of costs, choice of drug, efficacy, side-effects and compliance. It is therefore to everyone's interest to improve prescribing. This study looked at some of the reasons behind choice of drug prescribed by psychiatrists in seven Manchester hospitals.

\section{The study}

Doctors of all ranks, from senior house officer to consultant, in seven of nine hospitals in the Manchester psychiatry scheme, were invited to participate in the study via letter or personally. Of the seven hospitals included, three were university hospitals and four district general hospitals. Two hospitals were not included because of logistic difficulties. Where possible a local doctor or secretary was asked to act as a focus distributing and collecting the questionnaire. The instrument used was a two-question survey. It asked about the drug of first choice in an uncomplicated first-episode schizophrenia, depression and mania; and second, to indicate in order of decreasing importance from one to seven the reason for their choice. The doctors were asked to complete these for each of the following age groups: under 18, 18-64 and 65 and over. Six possible choices were suggested. These were: personal experience, scientific evidence, ward or unit policy, economic consideration, influence from senior colleagues and encouragement from drug representatives. A seventh possibility was left blank to allow for any other reasons to be specified by each participant if they wished to. In addition, doctors were asked to indicate their speciality, rank and hospital.

\section{Findings}

Out of 130 questionnaires sent, 88 were returned giving a response rate of $68 \%$. The distribution of responders were 37 consultants, 17 senior registrars, 15 registrars and 19 senior house officers. Most doctors were general adult psychiatrists $(70 \%)$, but old age, alcohol and substance misuse, child and adolescent and rehabilitation psychiatrists also responded. The three most important influences on choice of prescription were personal experience, scientific evidence and influences by colleagues, in that order (Fig. 1). This remained true across the ranks, except for registrars who indicated scientific evidence was as important. The least important factors were economy and influence by drug representatives. This result was stable across all the ranks (Fig. 2).

\section{Comment}

Appropriate prescribing is a difficult task and is influenced by drug, patient and doctor factors. However, there is evidence to suggest that prescribing can be improved (Hogerzeil, 1995). This survey looked at some of the reasons that govern choice of drug. The idea behind this approach is that if we explore the process of prescribing, we will be better able to modify that process successfully. It is acknowledged that in practice the doctor will be faced with a patient who may have other confounding factors such

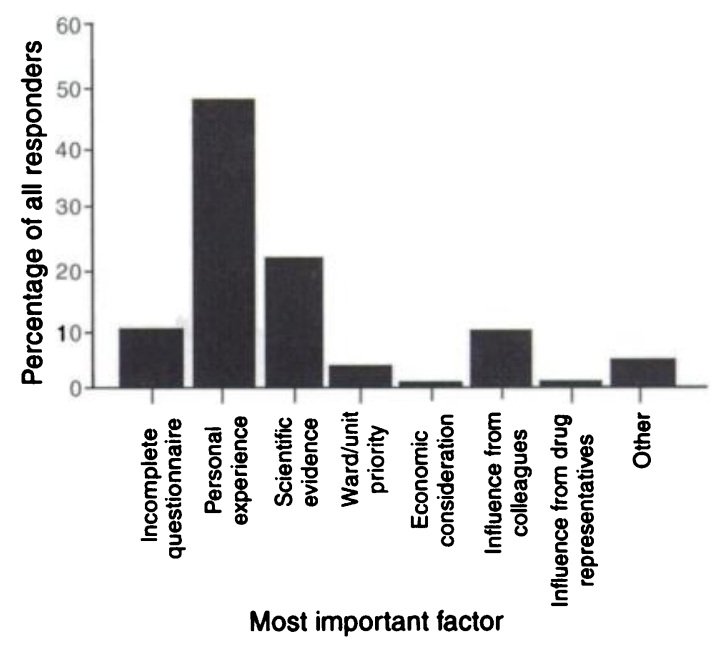

Fig. 1. Rationale behind prescribing among psychiatrists in Manchester (all doctors). 


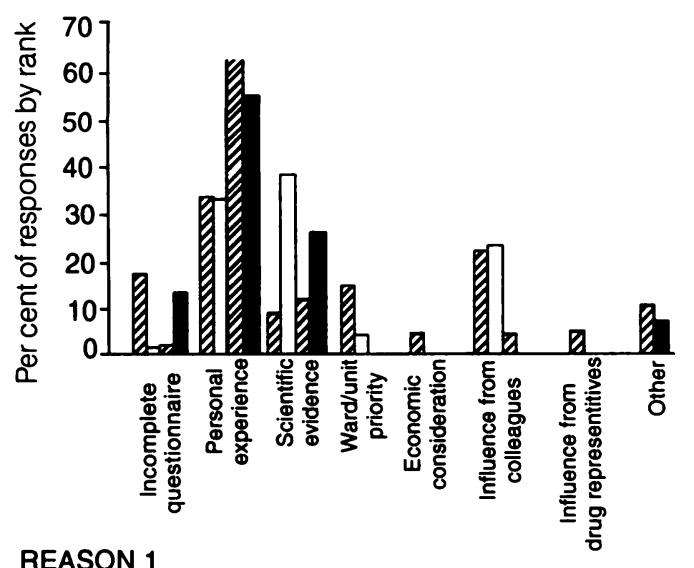

REASON 1

Most important factor

Fig. 2. Rationale behind prescribing amont psychiatrists in Manchester (percentage of responses by rank). senior house officer: $\square$,

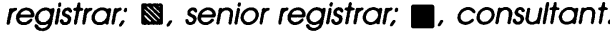

as, other medications or other illness. None the less, some idea of how medical staff choose a drug can be obtained from the above survey as it was the basis for the choice that was important here.

The principal finding was that personal experience was the most important factor in choosing a prescription in $50 \%$ of responses. Scientific evidence and influence by colleagues were second and third with 20 and $10 \%$, respectively. Economy and influence by drug representatives were the least important. In the present climate, so much is made of expensive drugs and treatments. It is therefore surprising that economic consideration should rank among the least important factors. Medical education does depend to some extent on observation and emulating teachers. Inevitably personal experience will mean different things to different doctors. A senior house officer or registrar will not have the same level of experience as a consultant, and even among consultant staff, such experiences are different. But while the personal approach has its place and some argue that medicine remains as much an art as it is a science, available evidence suggests the evidence-based consensus route may be better (Hogerzell, 1995; MacLeod, 1996). How the personal experience was obtained or what it meant was not assessed in this survey but it is clearly an important factor. It is therefore suggested that an understanding of this may be helpful in improving prescribing.
The other finding in this survey, as in other studies, is that influence from drug representatives was not important in deciding what to prescribe. This is at odds with commercial sense on the part of the pharmaceutical industry and also with research finding (Lexchin, 1993). Why would drug companies spend so much on employing representatives if they have no influence on doctors prescribing habits? Could it be that they are ahead of the game and know how to equip doctors with this thing we call 'personal experience'?. In general practice, in Canada and UK at least, drug representatives were the first or second most frequently used source of drug information. It has been shown that following expenses paid-trips, prescriptions of the sponsors drug increase. A similar result has also been seen with increased contact with the drug representative. Drug companies may interact with doctors at three different levels: research funding; continuing medical education sponsorship and, of course, the visiting drug representative. While it remains uncertain whether the first two interactions are beneficial or detrimental to good prescribing, there is evidence to suggest that the last interaction can be detrimental (Lexchin, 1993). In a previous survey, Macpherson \& Robson (1994) excluded pre-MRCPsych registrars as they were felt to be a different group. The present study appears to support this with regard to their basis for prescribing. They were the only group to cite scientific evidence as the most important influence. What was peculiar about this group is most of them were in preparation for the MRCPsych examination. This may be a significant factor. This is an observation worthy of further investigation. The results, however, should be interpreted with caution on several counts as the questionnaire used in the study has not been validated and not been subjected to reliability studies. It is however reassuring that the findings echo those of other investigators (Lexchin, 1993). A second question is to what extent do the self responses represent actual practice. Perhaps a prospective design would answer such a question. A further issue is just how representative of clinical practice were the study sample? The study sample was drawn from both university hospitals as well as district general hospitals within the Greater Manchester scheme. While it is tempting to assume that psychiatrists or indeed doctors in other areas may behave in a similar way, only a multi-centre study would clearly support or refute such an assumption. Lastly, $32 \%$ of the questionnaires were not returned. It is therefore uncertain how this may have affected the results.

Whatever the merits or demerits of personal experience, it seems clear that as prescribers, psychiatrists trust mostly in their own experiences. It is not likely that this is peculiar to 
Manchester or indeed psychiatrists. An understanding of the way in which this personal experience is obtained and maintained may help improve rational prescribing. One source of this experience is the pharmaceutical industry whose influence may be greater than we realise or are prepared to acknowledge. In acknowledging the possible influences of the pharmaceutical industry both positive and negative, we may be better able to judge how best to avoid prescribing pitfalls. As suggested by Hogerzeil (1995), the future prescriber should be equipped with problem-solving. Problem-based learning is now taking root in a number of medical schools including Manchester. It will therefore be interesting to see the impact of this on future prescribing. Basing prescribing decisions on personal experience is not necessarily a bad thing but ensuring that the right experience is obtained is important.

\section{Acknowledgements}

Thanks to all those who participated in the survey. I am grateful to Dr G. Rait, Dr C. Jagus and $\mathrm{Mr} \mathrm{L}$. Furniss for valuable discussions on the questionnaire; Dr E. Russell, Dr A. Juhasz, Dr P. Marshall, Mrs S. Morgan, Mrs J. Gibson,
Ms A. Day and Ms B. Woodyat for distributing the questionnaire and Dr E. J. Byrne, Dr C. J. M. Chithila and Professor A. Burns for their helpful suggestions.

\section{References}

Bateman, D. N.. Eccles, M., Campbell, M., et al (1996) Setting standards of prescribing performance in primary care: use of a consensus group of general practitioners and application of standards to practices in the north of England. British Journal of General Practice, 46, 20-25.

HoGerzeIL, H. V. (1995) Promoting rational prescribing: an international perspective. British Journal of Clinical Pharmacology, 39, 1-6.

LEXCHIN, J. (1993) Interactions between physicians and the pharmaceutical industry: What does the literature say? Canadian Medical Association Journal. 149. 1401-1406.

MACLEOD, S. M. (1996) Improving physician prescribing practices: bridge over troubled waters. Canadian Medical Association Journal, 164, 675-677.

MACPHERSON, R. \& ROBSON, E. (1994) How do clinicians choose antidepressants? Psychiatric Bulletin. 18, 597599.

John Dickson-Mulinga, Senior Registrar in Psychiatry, Withington Hospital, Nell Lane, West Didsbury, Manchester M20 8LR

\title{
Prescriptions, licences and evidence
}

\author{
David Healy and David Nutt
}

\begin{abstract}
Aims and method There is considerable confusion at present among clinicians as regards the appropriateness of prescribing off-licence. Because of the nature of the registration process it is likely that a considerable proportion of prescribing will always be off-licence. This paper seeks to clarify when it is appropriate to prescribe off-licence. We convened a workshop on behalf of the British Association for Psychopharmacology involving clinicians and regulators from a variety of countries to explore this issue both generally and for specific childhood and learning disability clinical situations. Recent statements from the defence unions and consumer groups were also scrutinised.
\end{abstract}

Results Across senior clinicians and regulators from a number of European countries and North America, there is a consensus that prescribing off-licence is a necessary part of the art of medicine.

Clinical implications Current advice to clinicians on the issue of off-licence prescribing can sometimes overemphasise the hazards and neglect the benefits that may stem from appropriate off-licence prescribing. Good prescribing involves specifying treatment goals and monitoring outcomes and it is more important to share this with the patient than it is to communicate the licensed status of the drug being prescribed. 